PROCEEDINGS OF THE AMERICAN MATHEMATICAL SOCIETY

Volume 124, Number 5, May 1996

\title{
THE MODULAR GROUP ALGEBRA PROBLEM FOR METACYCLIC $p$-GROUPS
}

\author{
ROBERT SANDLING
}

(Communicated by Ronald M. Solomon)

\begin{abstract}
It is shown that the isomorphism type of a metacyclic $p$-group is determined by its group algebra over the field $F$ of $p$ elements. This completes work of Bagiński. It is also shown that, if a $p$-group $G$ has a cyclic commutator subgroup $G^{\prime}$, then the order of the largest cyclic subgroup containing $G^{\prime}$ is determined by $F G$.
\end{abstract}

The isomorphism problem for modular group algebras asks whether a finite $p$ group is determined up to isomorphism by its group algebra over a field of characteristic $p$. It has been most successfully answered for classes of $p$-groups which are recognisable from the information known to be contained in the group algebra and which admit a complete classification (e.g., abelian $p$-groups, groups of order $p^{4}$ ). A metacyclic group is one which is the extension of one cyclic group by another. Metacyclic $p$-groups have received a plethora of classifications (see $[2,4,9,7]$ and their bibliographies), with more in the offing: [8]. They comprise a class recognisable in the sense above; moreover, all the information needed to determine the individual groups is given by invariants known to be determined by a modular group algebra. The isomorphism problem thus has a positive answer in their case. The object of this note is the presentation of the details of the argument. Our work completes that of Bagiński [1] who proved the theorem for $p \geq 5$. It also simplifies it by making use of different invariants and of a different classification, that of King [4] which seems the most amenable to the purpose.

Throughout, $p$ will denote a fixed prime and $F$ a field of characteristic $p$, often the field of $p$ elements. $G$ will be a finite $p$-group with $F G$ its modular group algebra. The terms of its lower central series will be denoted by $G_{n}$ (its commutator subgroup will also be denoted by $G^{\prime}$ ).

The invariants of $G$ which we need are few: its order, the isomorphism types of its commutator factor group, of its centre and of its section $G / \Phi\left(G^{\prime}\right) G_{3}$, the number of conjugacy classes of maximal elementary abelian subgroups of each rank, the number of conjugacy classes consisting of $p^{n}$-th powers for each $n \geq 0$. All but the last were mentioned in my survey $[10, \S 6]$. Most, but not all, of these invariants are known to be determined by $F G$ for any field $F$ of characteristic $p$.

Received by the editors July 11, 1994.

1991 Mathematics Subject Classification. Primary 20C05; Secondary 16S34, 16U60, 20C20, 20D15, 20F05.

Key words and phrases. Modular group algebra, p-group, isomorphism problem, metacyclic.

(c)1996 American Mathematical Society 
That the last is an invariant, is a result of Külshammer (see [5]) who brought it to my attention, admonishing me for its omission from my survey. He has given another exposition in his survey [6]. Part of the result can also be found in [13]. An important consequence of the result is the fact that $F G$ determines the exponent of $G$ [5, Satz J]. This has also been shown by Shalev by a different technique [12]. The precise statement of the result is as follows.

Proposition 1. Let $G$ be a finite p-group and $F$ a field of characteristic $p$. Then, for each $n \geq 0$, the number of conjugacy classes of $G$ of elements of the form $g^{p^{n}}$, $g \in G$, is determined by FG. Consequently, the exponent of $G$ is so determined.

Proof. The elements of $F G$ whose $p^{n}$-th powers lie in the subspace $(F G, F G)$ of all Lie commutators, form a subspace of $F G$ denoted as $T_{n}(F G)$ in [5] (the quotient space $F G /(F G, F G)$ may be viewed as a commutative restricted Lie algebra). The number in question, plus the dimension of $T_{n}(F G)$ (pace [6]), is the order of $G$.

Beyl's thesis demonstrated the importance of cyclic subgroups of $G$ which are of maximal order subject to containing the commutator subgroup $G^{\prime}$. This order is also an invariant obtainable from $F G$; an alternative proof of our main theorem may be given by using it and the classification in [2]. We prove this fact here as a further contribution to the isomorphism problem, one which may be relevant to showing that groups close to being metacyclic are also determined by their modular group algebras.

Definition 2. Let $G$ be a finite $p$-group. If its commutator subgroup $G^{\prime}$ is cyclic, let $B(G)=\max \left\{\log _{p}|N|: G^{\prime} \leq N \leq G, N\right.$ cyclic $\}$; if not, let $B(G)=-\infty$.

Lemma 3. Let $G$ be a finite p-group whose commutator subgroup is cyclic. If the normal subgroup $L$ of $G$ is properly contained in $G^{\prime}$, then $B(G)=B(G / L)+B(L)$.

Proof. We will use the bar convention to denote images in the quotient group $\bar{G}=$ $G / L$. Suppose that $N, G^{\prime} \leq N \leq G$, is such that $\bar{N}$ is cyclic with $\log _{p}|\bar{N}|=B(\bar{G})$. As $\bar{G}^{\prime}=\overline{G^{\prime}}$ is nontrivial, $L \leq \Phi\left(G^{\prime}\right)$, the Frattini subgroup of $G^{\prime}$, so that $N$ is itself cyclic. Thus $B(G) \geq \log _{p}|N|=\log _{p}|\bar{N}|+\log _{p}|L|=B(\bar{G})+B(L)$.

For the converse let $M$ be a cyclic subgroup of $G$ containing $G^{\prime}$. As $\bar{M}$ is cyclic, $\log _{p}|\bar{M}| \leq B(\bar{G})$. Thus $\log _{p}|M| \leq B(\bar{G})+B(L)$ from which it follows that $B(G) \leq B(\bar{G})+B(L)$.

Proposition 4. Let $G$ be a finite p-group and $F$ the field of $p$ elements. Then the invariant $B(G)$ is determined by $F G$.

Proof. Whether or not $B(G)=-\infty$, i.e., whether or not $G^{\prime}$ is cyclic, is determined by $F G$ according to the argument following Lemma 6.26 of [10]. Assume then that $B(G) \geq 0$. As $G^{\prime}$ is cyclic, $\Phi\left(G^{\prime}\right)=G_{2}^{p} G_{3}$ so that $F G$ determines $G / \Phi\left(G^{\prime}\right)$ [11]. If the latter is $G$, then $B(G)$ is also determined. If not, then $B\left(G / \Phi\left(G^{\prime}\right)\right)$ is determined. As $\left|G^{\prime}\right|$ is determined $[10,6.12]$, so are $\left|\Phi\left(G^{\prime}\right)\right|$ and $B\left(\Phi\left(G^{\prime}\right)\right)=$ $\log _{p}\left|\Phi\left(G^{\prime}\right)\right|$. By the previous lemma $B(G)=B\left(G / \Phi\left(G^{\prime}\right)\right)+B\left(\Phi\left(G^{\prime}\right)\right)$ so that $B(G)$ is itself determined.

All the preliminaries being to hand, we may now state and prove our main theorem.

Theorem 5. Let $G$ be a metacyclic p-group and $F$ the field of $p$ elements. Then the isomorphism type of $G$ is determined by $F G$. 
Proof. Whether or not $G$ is metacyclic is determined by $F G$; this follows from a result of Shalev [12], or from the facts that $G$ is metacyclic if and only if $G / \Phi\left(G^{\prime}\right) G_{3}$ is metacyclic [3, III.11.3] and that the latter group is determined by $F G$ [11].

By [4, Theorem 3.3] there is at most one metacyclic $p$-group $G$ of given order, commutator factor group and centre unless, for some $n \geq 1, G / G^{\prime} \cong C_{2^{n}} \times C_{2}$ and $\zeta(G) \cong C_{2^{n-1}} \times C_{2}$. These are invariants of $G$ which are determined by $F G[10$, $6.12,6.7]$.

It remains to distinguish the 2 -groups which arise in the latter case. When $n=1$ these are the 2-groups of maximal class [3, III.11.9]. They have been known to be distinguished by their modular group algebras for a long time [10, 6.34]. Another approach can be based on an invariant of Leonard Scott reported in [10, 6.28]. For these groups it amounts to the easy calculation of the number of conjugacy classes of elementary abelian subgroups of rank 2, as was done for the groups of order 64 in [13].

When $n>1$ there are precisely two groups of the same order which arise; for $m \geq 3$ :

$$
\left\langle a, b \mid a^{2^{m}}=1=b^{2^{n}}, a^{b}=a^{-1}\right\rangle ; \quad\left\langle a, b \mid a^{2^{m}}=1=b^{2^{n}}, a^{b}=a^{-1+2^{m-1}}\right\rangle .
$$

They may be distinguished by Külshammer's invariants (as was done in [13] in the order 64 case). The numbers of conjugacy classes of squares differ. In both of these groups the subsets $\left\{b^{4 i} a^{2 j}, b^{4 i} a^{-2 j}\right\}, i, j$ arbitrary, and $\left\{b^{2 i}\right\}, i$ odd, are conjugacy classes. In the first case these are the only conjugacy classes of squares while in the second case there are additionally the central subsets $\left\{b^{2 i} a^{2^{m-1}}\right\}, i$ odd.

\section{ACKNOWLEDGEMENTS}

I wish to thank Rudi Beyl and Mike Newman for calling the work of Bruce King to my attention, and to Geoff Smith's group-pub-forum for further bibliographic information.

\section{REFERENCES}

1. C. Bagiński, The isomorphism question for modular group algebras of metacyclic p-groups, Proc. Amer. Math. Soc. 104 (1988), 39-42. MR 89i:20016

2. F. R. Beyl, The classification of metacyclic p-groups, and other applications of homological algebra to group theory, Ph. D. thesis, Cornell Univ., 1972.

3. B. Huppert, Endliche Gruppen I, Springer, Berlin, 1967. MR 37:302

4. B. W. King, Presentations of metacyclic groups, Bull. Austral. Math. Soc. 8 (1973), 103-131. MR 48:2246

5. B. Külshammer, Bemerkungen über die Gruppenalgebra als symmetrische Algebra, II, J. Algebra 75 (1982), 59-69. MR 83j:16017b

6. _ Group-theoretical descriptions of ring-theoretical invariants of group algebras, Prog. in Math. 95 (1991), 425-442. MR 92d:16037

7. S. Liedahl, Presentations of metacyclic p-groups with applications to K-admissibility questions, J. Algebra 169 (1994), 965-983. CMP 95:03

8. M. F. Newman and M. Xu, Metacyclic groups of prime-power order, Adv. Math. (Beijing) 17 (1988), 106-107.

9. L. Redéi, Endliche p-Gruppen, Akadémiai Kiadó, Budapest, 1989. MR 90i:20001

10. R. Sandling, The isomorphism problem for group rings: a survey, Orders and their applications (Oberwolfach, 1984), 256-288, Lecture Notes in Mathematics 1142, Springer, Berlin, 1985. MR 87b:20007

11. (Basel) 52 (1989), 22-27. MR 90b:20007 
12. A. Shalev, Dimension subgroups, nilpotency indices, and the number of generators of ideals, J. Algebra 129 (1990), 412-438. MR 91a:20011

13. M. Wursthorn, Die modularen Gruppenringe der Gruppen der Ordnung $2^{6}$, Diplomarbeit, Universität Stuttgart, 1990.

Department of Mathematics, The University, Manchester M13 9PL, England

E-mail address: rsandling@manchester.ac.uk 